PROCEEDINGS OF THE

AMERICAN MATHEMATICAL SOCIETY

Volume 29, Number 3, August 1971

\title{
WALLMAN-TYPE COMPACTIFICATIONS AND PRODUCTS ${ }^{1}$
}

\section{FRANK KOST}

Abstract. $Y$ is a Wallman-type compactification (O. Frink, Amer. J. Math. 86 (1964), 602-607) of $X$ in case there is a normal base $Z$ for the closed sets of $X$ such that the ultrafilter space from $Z$, denoted $\omega(Z)$, is topologically $Y$. It is not known if every compactification is Wallman-type. For $Z_{\alpha}$ a normal base for the closed sets of $X_{\alpha}$ for each $\alpha$ belonging to an index set $\Delta$ it is shown that the Tychonoff product space $\prod_{\alpha \in \Delta} \omega\left(Z_{\alpha}\right)$ is a Wallman compactification of $\prod_{\alpha \in \Delta} X_{\alpha}$. Also for $X \subset T \subset \omega(Z)$ with $Z$ a normal base for the closed sets of $X$, a proof that $\omega(Z)$ is a Wallman-type compactification of $T$ is indicated.

Introduction. In 1938, H. Wallman [5] associated with a $T_{1}$-space $X$ a compact $T_{1}$-space $S$ which contained a dense copy of $X$. In this construction the points of $S$ are the ultrafilters from the lattice of closed subsets of $X$. O. Frink [2] characterized complete regularity in $T_{1}$-spaces as those that possess a normal base for their closed sets. In the proof of sufficiency he constructed a Hausdorff compactification $\omega(Z)$ of $X$ by considering the ultrafilters from the normal base $Z$. As this construction was utilized by Wallman, Frink called a compactification obtained in this way a Wallman-type compactification and offered the conjecture that every compactification is Wallmantype. This question is unsettled.

The Stone-Cech compactification of $X$ [3] and the one-point compactification of locally compact $X$ [1] are Wallman-type. Sufficient conditions on a compact space are known that insure that it is a Wallman-type compactification of each of its dense subspaces [4]. Every well-ordered set with the last element endowed with the order topology enjoys these conditions. Hence the set of ordinal numbers less than or equal to the first uncountable one provides an example of a Wallman-type compactification of an uncountable discrete space (the nonlimit ordinals).

It is natural to ask if the Tychonoff product space $\Pi \omega\left(Z_{\alpha}\right)$ is a Wallman-type compactification of $\prod X_{\alpha}$ where $\omega\left(Z_{\alpha}\right)$ is a Wallman-

Received by the editors October 1, 1970.

$A M S 1970$ subject classifications. Primary 54D35; Secondary 54C50, 54C25.

Key words and phrases. Wallman-type compactification, normal base, zero set, free ultrafilter.

1 This research was supported in part by the State University of New York Research Foundation under contract number 0966-03-027-69-0. 
type compactification of $X_{\alpha}$ for each $\alpha \in \Delta$ and $\Delta$ an index set of arbitrary cardinal. We show that it is.

Definitions. $Y$ is a compactification of $X$ if $Y$ is a compact Hausdorff space and a copy of $X$ is dense in $Y$. A family $Z$ of closed sets of a space $X$ is said to be

(i) a base for the closed sets of $X$ if $x \notin A, A$ closed, there is $F \in Z$ with $x \notin F$ and $A \subset F$;

(ii) a ring of sets if $Z$ is closed under the formation of finite intersections and unions;

(iii) disjunctive if for $x \notin A, A$ closed, there is $F \in Z$ with $x \in F$ and $F \cap A=\varnothing$;

(iv) a normal family if, whenever $F_{1}, F_{2} \in Z$ with $F_{1} \cap F_{2}=\varnothing$, there is $H_{1}, H_{2} \in Z$ with $F_{1} \subset X \backslash H_{1}, F_{2} \subset X \backslash H_{2}$ and $\left(X \backslash H_{1}\right) \cap\left(X \backslash H_{2}\right)=\varnothing$, i.e., complements of members of $Z$ separate disjoint members of $Z$.

In case $Z$ satisfies (i) $\rightarrow$ (iv) above $Z$ is called a normal base for the closed sets of $X$. The lattice of all closed sets of a $T_{1}$-space $X$ possesses (i) $\rightarrow$ (iii) and is a normal base if and only if $X$ is a normal space. The collection of finite unions of sets of the form $(0, b]$, $[a, 1),[a, b]$ with $0<a, b<1$ is a normal base for the closed sets of $(0,1)$ with the usual topology. For $X$ a discrete space we have $\{F \subset X: F$ finite or $X \backslash F$ finite $\}$ is a normal base. In case $X$ is completely regular- $T_{1}\left(T_{3 \frac{1}{2}}\right)$ the family of zero-sets of continuous realvalued functions on $X$ is a normal base. For $Z$ a ring of closed sets that is a disjunctive base for the closed sets of $X$ we set $\omega(Z)$ $\equiv\{a: a \subset Z, a$ an ultrafilter $\}$. The ultrafilters from $Z$ with nonempty intersection correspond to the points of $X$ and those with empty intersection (free ultrafilters) are the points that are "added" to $X$ to obtain $\omega(Z)$. If $X$ is compact there are no free ultrafilters and no points to be "added." $\omega(Z)$ is topologized as follows: for $F \in Z$ define $F^{*} \equiv\{a \in \omega(Z): F \in a\}$. As $Z$ is closed under finite unions, $\left\{F^{*}: F \in Z\right\}$ is a base for the closed sets of some topology on $\omega(Z)$. $\omega(Z)$, with this structure, is the compact $T_{1}$-space of our concern.

LEMMA 1. Let $Z$ be a ring of closed sets that is a disjunctive base for the closed sets of $X . \omega(Z)$ is a Hausdorff space if and only if $Z$ is a normal family.

Proof. The sufficiency appears in [2] and is basic to the conjecture. Now assume $\omega(Z)$ is Hausdorff. For $F_{1}, F_{2} \in Z$ with $F_{1}, F_{2}$ disjoint, it follows that $F_{1}^{*} \cap F_{2}^{*}=\varnothing . \omega(Z)$ Hausdorff implies $\omega(Z)$ is normal since it is also compact. As a result $F_{1}^{*}$ and $F_{2}^{*}$ can be separated by 
basic open sets $O_{1}$ and $O_{2}$ in $\omega(Z)$. The traces of $O_{1}$ and $O_{2}$ in $X$ separate $F_{1}$ and $F_{2}$ and their complements belong to $Z$.

The collection of finite unions from $(0, b],[a, 1),[a, b]$ with $0<a$, $b<1$ yields the 2 -point compactification $([0,1])$ of $(0,1)$. For $X$ discrete and $Z=\{F \subset X: F$ finite or $X \backslash F$ finite $\}$, the space $\omega(Z)$ is the one-point compactification of $X$, i.e., there is just one free ultrafilter from $Z$. If $X$ is $T_{3 \frac{1}{2}}, Z=$ the zero sets of real valued continuous functions on $X$, Gillman and Jerison [3] have shown that $\omega(Z) \approx \beta(X)$ where $\beta X$ is the Stone-Cech compactification of $X$. Frink's conjecture can be rephrased as follows: If $Y$ is a compactification of $X$ does there exist a normal base $Z$ for the closed sets of $X$ with $\omega(Z) \approx Y$.

For $Z_{\alpha}$ a normal base for the closed sets of $X_{\alpha}$, for each $\alpha \in \Delta$, we can form $\omega\left(Z_{\alpha}\right)$. The cartesian product space $\prod_{\alpha \in \Delta} \omega\left(Z_{\alpha}\right)$ is a compactification of $\prod_{\alpha \in \Delta} X_{\alpha}$ which, as we proceed to show, is Wallmantype. First we need a lemma.

LEмма 2. Take $F$ a family of sets closed under finite intersection. There is a 1-1 correspondence between the ultrafilters from $\mathcal{F}$ and those from $\mathcal{F}_{\Sigma}$ where $\mathcal{F}_{\Sigma}$ is all finite unions from $\mathcal{F}$.

Proof. The correspondence we obtain will be $a \rightarrow J_{a}$ where $a$ is an ultrafilter from $\mathcal{F}$ and $J_{\mathbb{Q}}$ is the unique ultrafilter from $\mathcal{F}_{\Sigma}$ that contains $a$. First note that distinct ultrafilters from $\mathcal{F}$ can be embedded in distinct ultrafilters from $\mathcal{F}_{\Sigma}$. Now let $J$ be an ultrafilter from $\mathcal{F}_{\Sigma} . J=\left\{F_{\alpha}\right\}_{\alpha \in \Delta}$ with $F_{\alpha}=\bigcup_{i=1}^{n} A_{\alpha_{i}}, A_{\alpha_{i}} \in \mathcal{F}$ for each $\alpha \in \Delta$. J maximal implies $J$ prime so for some $i_{0}, 1 \leqq i_{0} \leqq n, A_{\alpha_{i}} \in J$. Denote $A_{\alpha_{i 0}}$ by $A_{\alpha}$ and observe that $\left\{A_{\alpha}\right\}_{\alpha \in \Delta}$ has the Finite Intersection Property in $\mathcal{F}$, so it can be embedded in an ultrafilter $a$ from $\mathcal{F}$. If $A \in Q$ then $A \cap A_{\alpha} \neq \varnothing$ for every $\alpha \in \Delta$ and since $A_{\alpha} \subset F_{\alpha}$ we have $A \cap F_{\alpha} \neq \varnothing$ for every $\alpha \in \Delta$. Since $A$ intersects every member of $\mathrm{J}$ it follows that $A \in J$ and $a \subset J$. To see uniqueness let $J_{1}$ and $J_{2}$ be ultrafilters from $\mathcal{F}_{\Sigma}$ each containing $a$. Assume $J_{1} \neq J_{2}$ and take $F \in J_{2}$ with $F \notin J_{1} . F=\bigcup_{i=1}^{n} A_{i}, A_{i} \in \mathcal{F}$ and $J_{2}$ prime implies there is $i_{0}, 1 \leqq i_{0} \leqq n$, with $A_{i_{0}} \in J_{2}$. Now $A_{i_{0}}$ intersects every member of $a\left[a \subset J_{2}\right]$ so we have $A_{i_{0}} \in Q$. There is $F_{1} \in J_{1}$ with $F_{1} \cap F=\varnothing$ and since $A_{i_{0}} \subset F$ it follows that $A_{i_{0}} \cap F_{1}=\varnothing$, i.e., $A_{i_{0}} \notin J_{1}$ which contradicts our assumption that $a \subset J_{1}$. Therefore $J_{1}=J_{2}$. In fact, $J_{a} \cap \mathcal{F}=Q$.

Theorem. Let $Z_{\alpha}$ be a normal base for the closed sets of $X_{\alpha}$ for each $\alpha \in \Delta . \prod_{\alpha \in \Delta} \omega\left(Z_{\alpha}\right)$ is a Wallman-type compactification of $\prod_{\alpha \in \Delta} X_{\alpha}$.

Proof. Let $Z \equiv\left\{\prod F_{\alpha}: F_{\alpha} \in Z_{\alpha}\right.$ and $F_{\alpha}=X_{\alpha}$ for all but a finite number of $\alpha \in \Delta\}$ and $Z_{\Sigma}$ be the collection of all finite unions from $Z$. 
The family $C$ of complements of members of $Z_{\Sigma}$ is a collection of open subsets of $\prod X_{\alpha}$ that contains $\left\{\prod_{\alpha \in \Delta} O_{\alpha}: O_{\alpha}=X_{\alpha}\right.$ for all but finitely many $\alpha, X_{\alpha} \backslash O_{\alpha} \in Z_{\alpha}$ for other $\left.\alpha\right\}$. Hence $C$ is a base for the open sets of $\prod X_{\alpha}$ and $Z_{\Sigma}$ is a base for the closed sets of $\prod X_{\alpha}$. To see that $Z_{\Sigma}$ is disjunctive take $x=\left\langle x_{\alpha}\right\rangle \notin A, A$ closed in $\prod X_{\alpha}$. Since $Z_{\Sigma}$ is a base there is $F \in Z_{\Sigma}$ with $\left\langle x_{\alpha}\right\rangle \notin F$ and $A \subset F$. Now $F=\bigcup_{i=1}^{n} A_{i}, A_{i} \in Z$, and for each $i, 1 \leqq i \leqq n, x=\left\langle x_{\alpha}\right\rangle \notin A_{i}=\prod_{\alpha \in \Delta} A_{i_{\alpha}}$ so there is $\alpha \in \Delta$ with $x_{\alpha} \notin A_{i_{\alpha}} . Z_{\alpha}$ is disjunctive so take $F_{i_{\alpha}} \in Z_{\alpha}$ with $x_{\alpha} \in F_{i_{\alpha}}$ and $F_{i_{\alpha}} \cap A_{i_{\alpha}}=\varnothing$. Let $B_{i}$ be the set $\prod_{\alpha \in \Delta} A_{i_{\alpha}}$ with $F_{i_{\alpha}}$ replacing $A_{i_{\alpha}}$. For each $i, x=\left\langle x_{\alpha}\right\rangle \in B_{i}$ and $B_{i} \cap A_{i}=\varnothing$ so we have $x \in \bigcap_{i=1}^{n} B_{i} \in Z$ and $\left(\bigcap_{i=1}^{n} B_{i}\right) \cap F=\varnothing$. That $Z_{\Sigma}$ is a ring of sets results from $Z$ being closed under finite intersections and from the finite distributive laws of sets. We have that $Z_{\Sigma}$ is a disjunctive base for the closed sets of $\prod X_{\alpha}$ and a ring of sets, so the ultrafilter space $\omega\left(Z_{\Sigma}\right)$ can be formed. To continue the proof we need

Lemma 3. There is a 1-1 correspondence between points in $\prod_{\alpha \in \Delta} \omega\left(Z_{\alpha}\right)$ and ultrafilters from $Z$.

Proof. Take $\left\langle a_{\alpha}\right\rangle \in \prod \omega\left(Z_{\alpha}\right)$ and define $\prod a_{\alpha} \equiv\left\{\prod A_{\alpha}: A_{\alpha} \in a_{\alpha}\right.$ and $A_{\alpha}=X_{\alpha}$ for all but finitely many $\left.\alpha \in \Delta\right\}$. It is easily seen that $\Pi a_{\alpha}$ is a filter from $Z$ and $\left\langle a_{\alpha}\right\rangle \neq\left\langle\Theta_{\alpha}\right\rangle$ implies $\Pi a_{\alpha} \neq \Pi \leftrightarrow_{\alpha}$. Now let $J$ be an ultrafilter from $Z$ and for $\gamma \in \Delta$ set $J_{\gamma}=\left\{F \in Z_{\gamma}\right.$ : there is $\Pi F_{\alpha} \in J$ with $\left.F_{\gamma}=F\right\}$, i.e., $J_{\gamma}$ is the collection of $\gamma$ th components of members of $J$. That $J_{\gamma}$ is a filter from $Z_{\gamma}$ is a consequence of the equality $\left(\prod A_{\alpha}\right) \cap\left(\prod B_{\alpha}\right)=\prod\left(A_{\alpha} \cap B_{\alpha}\right)$ for $A_{\alpha}, B_{\alpha}$ subsets of $X_{\alpha}$. To see that $\jmath_{\gamma}$ is maximal take a filter $a$ from $Z_{\gamma}$ with $\jmath_{\gamma} \subset a$. Take $A \in Q$ and let $\prod F_{\alpha}$ be any member of $\Im$ and define $F^{\prime}=\prod F_{\alpha}$ where $F_{\gamma}=A$. We wish to show that $F^{\prime}$ meets every member of $J$ in which case we have $F^{\prime} \in J$ and $F_{\gamma}=A \in J_{\gamma}$ and $J_{\gamma}$ is an ultrafilter. Let $\prod A_{\alpha} \in$ J. $F^{\prime} \cap\left(\prod A_{\alpha}\right)=\prod\left(F_{\alpha} \cap A_{\alpha}\right)$ where $F_{\gamma}=A$. Now $A \cap A_{\gamma} \neq \varnothing$ since $A_{\gamma} \in J_{\gamma} \subset Q$ and $Q$ is a filter. We have $\left(\prod F_{\alpha}\right) \cap\left(\prod A_{\alpha}\right) \neq \varnothing$ as both belong to I so $F_{\alpha} \cap A_{\alpha} \neq \varnothing$ for $\alpha \neq \gamma$. As a result $F^{\prime} \cap\left(\prod A_{\alpha}\right)$ $\neq \varnothing$. For $J$ an ultrafilter from $Z$ we have $J_{\gamma}$ an ultrafilter from $Z_{\gamma}$ for each $\gamma \in \Delta$ and the filter $\prod_{\gamma \in \Delta} J_{\gamma}$ from $Z$ contains $J$. Therefore $\prod_{\gamma \in \Delta} \mathfrak{I}_{\gamma}=\mathfrak{J}$ and the ultrafilters from $Z$ are precisely $\left\{\prod_{\alpha \in \Delta} a_{\alpha}\right\}_{\left\langle a_{\alpha}\right\rangle \in \Pi_{\alpha \in \Delta \omega}\left(Z_{\alpha}\right)}$.

Define $\theta: \prod \omega\left(Z_{\alpha}\right) \rightarrow \omega\left(Z_{\Sigma}\right)$ by $\theta\left(\left\langle a_{\alpha}\right\rangle\right)=J_{\amalg a_{\alpha}}$ where $J_{\amalg a_{\alpha}}$ is the ultrafilter from $Z_{\Sigma}$ that contains the ultrafilter $\prod a_{\alpha}$ from $Z$. By Lemmas 2 and $3, \theta$ is well defined, $1-1$, and onto. To complete the proof of Theorem 1 we need a final lemma.

Lemma 4. $\theta$ is a homeomorphism. 
Proof. To establish continuity of $\theta$ we take a basic closed set $F^{*} \subset \omega\left(Z_{\Sigma}\right)$ and show that $\theta^{-1}\left(F^{*}\right)$ is closed in $\prod \omega\left(Z_{\alpha}\right)$. Now $F \in Z_{\Sigma}$ so $F=\bigcup_{i=1}^{n} F_{i}, F_{i} \in Z$, and note that $F^{*}=\bigcup_{i=1}^{n}\left(F_{i}^{*}\right)$. Recall that $F^{*}$ is the collection of ultrafilters from $Z_{\Sigma}$ that contain $F$. Set $F_{i}=$ $\prod_{\alpha \in \Delta} A_{i_{\alpha}}, A_{i_{\alpha}} \in Z_{\alpha}$, and $\theta^{-1}\left(F^{*}\right)=\theta^{-1}\left(\bigcup_{i=1}^{n}\left(F_{i}^{*}\right)\right)=\bigcup_{i=1}^{n} \theta^{-1}\left(F_{i}^{*}\right)$. We wish to show $\theta^{-1}\left(F_{i}^{*}\right)=\prod\left(A_{i_{\alpha}}^{*}\right)$ which is a basic closed set in $\prod \omega\left(Z_{\alpha}\right)$. If $\left\langle a_{\alpha}\right\rangle \in \theta^{-1}\left(F_{i}^{*}\right)$ then $\theta\left(\left\langle Q_{\alpha}\right\rangle\right)=J_{\Pi a_{\alpha}} \in F_{i}^{*}$ which means $F_{i} \in J_{\Pi a_{\alpha}}$. Now $F_{\imath}=\prod A_{i_{\alpha}}$ so $\prod A_{i_{\alpha}} \in{J_{\Pi a_{\alpha}}}$ Since $\prod A_{i_{\alpha}} \in Z$ we have $\prod A_{i_{\alpha}}$ $\in \prod a_{\alpha}$, so by definition $A_{i_{\alpha}} \in a_{\alpha}$ and $a_{\alpha} \in A_{i_{\alpha^{*}}}^{*}$ Therefore $\left\langle a_{\alpha}\right\rangle$ $\in \prod\left(A_{i_{\alpha}}^{*}\right)$ and $\theta^{-1}\left(F_{i}^{*}\right) \subset \prod\left(A_{i_{\alpha}}^{*}\right)$. If $\left\langle a_{\alpha}\right\rangle \in \prod\left(A_{i_{\alpha}}^{*}\right)$ then $a_{\alpha} \in A_{i_{\alpha}}^{*}$ for each $\alpha \in \Delta$ and $A_{i_{\alpha}} \in Q_{\alpha}$ so $\prod A_{i_{\alpha}} \in \prod a_{\alpha}$. This implies $\prod A_{i_{\alpha}}$ $\in J_{\Pi a_{\alpha}}$ and $\Im_{\Pi a_{\alpha}} \in\left(\prod^{\alpha} A_{i_{\alpha}}\right)^{*}$. However $\Im_{\Pi a_{\alpha}}=\theta\left(\left\langle Q_{\alpha}\right\rangle\right)$ and we have $\left\langle Q_{\alpha}\right\rangle \in \Theta^{-1}\left(\left(\prod A_{i_{\alpha}}\right)^{*}\right)=\theta^{-1}\left(F_{i}^{*}\right)$. Therefore $\prod\left(A_{i_{\alpha}}^{*}\right) \subset \theta^{-1}\left(F_{i}^{*}\right)$ and $\theta^{-1}\left(F_{i}^{*}\right)=\prod\left(A_{i_{\alpha}}^{*}\right)$. As a result $\theta^{-1}\left(F^{*}\right)$ is a closed set in $\prod \omega\left(Z_{\alpha}\right)$. By applying $\theta$ to both sides of $\theta^{-1}\left(\left(\prod A_{i_{\alpha}}\right)^{*}\right)=\prod\left(A_{i_{\alpha}}^{*}\right)$ we obtain $\left(\prod A_{i_{\alpha}}\right)^{*}=\theta\left(\prod\left(A_{i_{\alpha}}^{*}\right)\right)$ and $\theta$ takes basic closed sets in $\prod \omega\left(Z_{\alpha}\right)$ to closed sets in $\omega\left(Z_{\Sigma}\right)$, i.e., $\theta$ is a closed map. We have established that $\theta$ is a homeomorphism so $\omega\left(Z_{\Sigma}\right)$ is Hausdorff since $\prod \omega\left(Z_{\alpha}\right)$ is. By Lemma $1, Z_{\Sigma}$ is a normal base for $\prod X_{\alpha}$ and as a result $\prod \omega\left(Z_{\alpha}\right)$ is a Wallman-type compactification of $\Pi X_{\alpha}$. This completes the proof of the theorem.

By taking finite products of Wallman-type compactifications of $N$, the countably infinite discrete space, a class of compactifications of $N$, are obtained. By the theorem each is Wallman-type. Similarly for any discrete $X$. Also $\prod \beta X_{\alpha}$ is a Wallman-type compactification of $\prod X_{\alpha}$.

For $\omega(Z)$ a Wallman compactification of $X,\left\{F^{*}: F \in Z\right\}$ is a normal base for the closed sets of $\omega(Z)$ and for $X \subset T \subset \omega(Z)$ the collection $Z_{T}=\left\{F^{*} \cap T: F \in Z\right\}$ is a normal base for the closed sets of $T$. For $p \in \omega(Z)$ define $a_{p} \equiv\left\{F^{*} \cap T: p \in F^{*}\right\}$. The ultrafilters from $Z_{T}$ are precisely $\left\{a_{p}: p \in \omega(Z)\right\}$. The free ultrafilters are those $a_{p}$ with $p \in T$. The mapping $\theta: \omega(Z) \rightarrow \omega\left(Z_{T}\right)$ by $\theta(p)=Q_{p}$ is a homeomorphism and $\omega(Z)$ is a Wallman-type compactification of $T$. Therefore $\prod \omega\left(Z_{\alpha}\right)$ is a Wallman compactification of $T$ for $\prod X_{\alpha} \subset T \subset \prod \omega\left(Z_{\alpha}\right)$.

As mentioned earlier the conjecture that every compactification is Wallman-type is unsettled. It is not known if all compactifications of $N$ are. We have removed a class of the pathological Tychonoff product spaces as candidates for a counterexample. The problem of finding a counterexample seems difficult as one would have to prove the nonexistence of an appropriate normal base. 


\section{REFERENCES}

1. R. M. Brooks, On Wallman compactifications, Fund. Math. 60 (1967), 157-173.

2. O. Frink, Compactifications and semi-normal spaces, Amer. J. Math. 86 (1964), 602-607. MR $29 \# 4028$.

3. L. Gillman and M. Jerison, Rings of continuous functions, University Series in Higher Math., Van Nostrand, Princeton, N.J., 1960. MR 22 \#6994.

4. E. F. Steiner, Wallman spaces and compactifications, Fund. Math. 61 (1967/68), 295-304. MR 36 \#5899.

5. H. Wallman, Lattices and topological spaces, Ann. of Math. (2) 39 (1938), 112126.

State University College, Oneonta, New York 13820 